\section{PTU-102 DEVELOPMENT OF A FOOD RELATED QUALITY OF LIFE QUESTIONNAIRE FOR PEOPLE WITH IBD}

${ }^{1} \mathrm{~L}$ Hughes*, ${ }^{2} \mathrm{JO}$ Lindsay, ${ }^{3} \mathrm{MC}$ Lomer, ${ }^{1} \mathrm{~S}$ Ayis, ${ }^{1} \mathrm{~L}$ King, ${ }^{1} \mathrm{M}$ Morgan, ${ }^{1} \mathrm{~K}$ Whelan. ${ }^{1}$ King's College London, UK; ${ }^{2}$ Gastroenterology, Barts Health NHS Trust, London, UK; ${ }^{3}$ Nutrition and Dietetics, Guy's and St Thomas' Foundation NHS Trust, London, UK

\subsection{6/gutjnl-2014-307263.176}

Introduction The role of diet as a therapy for Inflammatory Bowel Disease (IBD) is well established and has improved treatment and prognosis for patients. However, little is understood about how IBD impacts on food-related quality of life. A qualitative study indicated a wide range of food related issues across a broad spectrum of IBD experiences which include, amongst others; identifying and avoiding trigger foods, uncertainty about eating and drinking, problems eating out and frustration around eating. This study aimed to develop a food-related quality of life (FRQoL) questionnaire for people with IBD.

Methods Semi-structured interviews with 28 IBD patients were coded and $150 \mathrm{FRQoL}$ questionnaire items were generated. One hundred IBD patients ranked each item on i) if it had been relevant to them in the past two weeks and; 2) how important it was to them (regardless of whether it had been relevant). Items were removed based on ceiling/floor effects and high inter-item correlations. The 41 highest ranking items were retained with a 5 point Likert (disagreeagree) response scale. Subsequently, 287 IBD patients, 100 asthma patients (chronic disease control) and 117 healthy volunteers completed the FRQoL questionnaire alongside clinical measures, MUST nutritional screening, food satisfaction and generic and disease-specific quality of life questionnaires. Psychometric testing of the FRQoL questionnaire has been carried out including principle components analysis, construct and discriminant validity and test-retest reliability. Results Initial principle components analysis identified seven components explaining $68.35 \%$ of variance with high internal reliability (Cronbach's alpha $=0.96$ ). The FRQoL sumscore (higher scores indicate worse quality of life) correlated with younger age $(r=-0.12)$, higher MUST score $(r=0.17)$ and lower BMI $(r=-0.13)$. Worse FRQoL was associated with female gender $(\mathrm{p}<0.001)$, a diagnosis of Crohn's Disease $(\mathrm{p}<$ $0.05)$, surgery $(\mathrm{p}<0.05)$ and high MUST score $(\mathrm{p}<0.05)$.

Conclusion An FRQoL questionnaire has been developed to identify issues around food, eating and nutrition for people with IBD at all stages of the disease process. Preliminary psychometric testing indicates that the questionnaire sumscore is related to clinical characteristics indicative of poor FRQoL. Further testing will determine the validity and reliability of the questionnaire for clinical use to identify IBD patients who may require further support with eating and drinking.

Disclosure of Interest None Declared.

\section{PTU-103 INTRA-LUMINAL INTERLEUKIN (IL)-27 IS A POTENTIAL FUTURE THERAPEUTIC FOR INFLAMMATORY BOWEL DISEASE}

${ }^{1}$ MH McLean*, ${ }^{1}$ ML Hanson, ${ }^{2} B$ Gold, ${ }^{3} Y$ Golubeva, ${ }^{3}$ MR Anver, ${ }^{4} X$ Wu, ${ }^{4} \mathrm{D}$ Sun, ${ }^{5} \mathrm{~L}$ Steidler, ${ }^{1} \mathrm{SK}$ Durum. ${ }^{1}$ Cancer and Inflammation Program; 'Human Genetics Section, National Cancer Institute; ${ }^{3}$ Lab Animal Sciences Program; ${ }^{4}$ Lab of Molecular Technology, Leidos Biomedical Research, Inc, Frederick National Laboratory for Cancer Research, Frederick, USA; ${ }^{5}$ ActoGenix N. V, Zwijnaarde, Belgium

\subsection{6/gutjnl-2014-307263.177}

Introduction Oral Lactococcus lactis engineered to express the immunoregulatory cytokine IL-27 (LL-IL27) is therapeutically active in chronic murine enterocolitis. Here, efficacy in acute colitis was examined.

Methods $2 \mathrm{mg}$ 2,4,6-trinitrobenzene sulfonic acid (TNBS) was delivered intra-rectally in $45 \%$ ethanol or $45 \%$ ethanol alone into 6-8 week old male SJL mice. L. lactis control (LLC) or LL-IL-27 was delivered by oral gavage on 4 occasions, $24 \mathrm{~h}$ apart, commencing at colitis induction. Therapeutic effect was assessed clinically and histologically. Potential mechanisms of action were investigated.

Results TNBS induced an acute severe distal colitis. LL-IL-27 led to a significant reduction in disease activity index compared to LLC (4.9 vs. $8.7 / 12$ on day 2 ( $\mathrm{p}=0.001)$; 3.6 vs. $7.7 / 12$ on day $3(\mathrm{p}=0.001)$, improved macroscopic colitis score $(\mathrm{p}<$ $0.05)$, and reduction in serum CRP ( $\mathrm{p}=0.003$, day 2$)$. Histological colitis score was reduced $(\mathrm{p}=0.035)$ with significant improvement in mucosal ulceration $(\mathrm{p}=0.008)$. TNBS increased expression of distal colon Il6, Il1ß, Tnf, and Il10, assessed by RT-PCR, with no differential effect seen with LL-IL27. However, LL-IL-27 led to a significant reduction in IL-6 ( $\mathrm{p}$ $=0.002)$, IL-1 $\beta(\mathrm{p}=0.001)$ and TNF $(\mathrm{p}=0.014)$ protein assessed by ELISA. A significant reduction in colonic mucosal myeloperoxidase $^{+}$neutrophil infiltrate was seen in the LL-IL-27 group $(p=0.004)$, along with a significant decrease in the neutrophil chemoattractant CXCL2 $(\mathrm{p}<0.001)$. LPS induced CXCL2 gene and protein expression in macrophages was not inhibited by recombinant IL-27 in vitro, suggesting an indirect mechanism in vivo. Peri-ulceration distal colonic mucosa was isolated by laser capture microdissection and RNA applied to mouse Genome 4302.0 Affymetrix microarray. Principal component analysis grouped mice by treatment. 285 genes were differentially expressed $(>/<1.5$ fold change in expression plus $\mathrm{p}<$ 0.05 ) in the LL-IL27 group, including a striking down-regulation of mucosal humoral response genes, (for example, probe sets for IgA heavy chain (-20.6 fold), Igא chain var1 (-19.0 fold), $\operatorname{Ig} \lambda$ chain CR2 (-4.8 fold)). This was not explained by a reduction in $\mathrm{CD} 45 \mathrm{R} / \mathrm{B} 220^{+} \mathrm{B}$ cell infiltrate $(\mathrm{p}=0.02)$. Up-regulated genes include those involved with anti-microbial defense (RegIIIb, Clec7A, ligp1) and innate immune response (cxcl10, cxcl9).

Conclusion Intra-luminal IL-27 represents a potential therapy for human inflammatory bowel disease and acute colitis of differing aetiologies.

Disclosure of Interest M. McLean: None Declared, M. Hanson: None Declared, B. Gold: None Declared, Y. Golubeva: None Declared, M. Anver: None Declared, X. Wu: None Declared, D. Sun: None Declared, L. Steidler Employee of: ActoGenix, S. Durum: None Declared.

\section{PTU-104 OUTCOMES OF INCREASED DOSE OF ALLOPURINOL IN IBD PATIENTS WHO DEVELOPED HEPATOTOXICITY ON LOW AZATHIOPRINE AND ALLOPURINOL CO-THERAPY TREATMENT}

M Pericleous*, A Abdulrehman, C Bull, G Mackenzie, J Stenner, M Cowan, G Hillman, A Ansari. Department of Gastroenterology, East Surrey Hospital, Surrey and Sussex Hospital NHS Trust, London, UK

\subsection{6/gutjnl-2014-307263.178}

Introduction The thiopurines (azathioprine (AZA) and metcaptopurine (6MP)) are established first line therapies for inflammatory bowel disease (IBD). However, when these agents are used at their target dose side effects are common, gastrointestinal intolerance (10-20\%) and hepatotoxicity (>10\%). ${ }^{1}$ These side effects can often be bypassed by using low dose AZA and 